\title{
Catheter Ablation of Atrial Fibrillation: State of the Art and Future Perspectives
}

\author{
Laura Rottner (D) - Barbara Bellmann - Tina Lin · Bruno Reissmann • \\ Tobias Tönnis · Ruben Schleberger • Moritz Nies • Christiane Jungen • \\ Leon Dinshaw · Niklas Klatt · Jannis Dickow · Paula Münkler • \\ Christian Meyer · Andreas Metzner · Andreas Rillig
}

Received: October 9, 2019 / Published online: January 2, 2020

(C) The Author(s) 2020

\begin{abstract}
Purpose of Review: Atrial fibrillation (AF), the most common sustained arrhythmia, is associated with high rates of morbidity and mortality. Maintenance of stable sinus rhythm (SR) is the intended treatment target in symptomatic patients, and catheter ablation aimed at isolating the pulmonary veins provides the most effective treatment option, supported by encouraging clinical outcome data. A variety of energy sources and devices have been developed and evaluated. In this review, we summarize the current state of the art of catheter ablation of $\mathrm{AF}$ and describe future perspectives. Recent Findings: Catheter ablation is a wellestablished treatment option for patients with
\end{abstract}

Enhanced Digital Features To view enhanced digital features for this article go to https://doi.org/10.6084/ m9.figshare.11357912.

L. Rottner $(\varangle) \cdot$ B. Reissmann · T. Tönnis .

R. Schleberger - M. Nies · C. Jungen - L. Dinshaw .

N. Klatt · J. Dickow · P. Münkler · C. Meyer .

A. Metzner · A. Rillig

Universitäres Herzzentrum Hamburg-Eppendorf,

Hamburg, Germany

e-mail: laurarottner@aol.de

B. Bellmann

MEDIAN Klinik AGZ Düsseldorf, Düsseldorf, Germany

T. Lin

GenesisCare, East Melbourne, VIC, Australia symptomatic $\mathrm{AF}$ and is more successful at maintaining SR than antiarrhythmic drugs. Antral pulmonary vein isolation (PVI) as a stand-alone ablation strategy results in beneficial clinical outcomes and is therefore recommended as first-line strategy for both paroxysmal and persistent AF. While radiofrequency-based PVI in conjunction with a threedimensional mapping system was for many years considered to be the "gold standard", the cryoballoon has emerged as the most commonly used alternative AF ablation tool, especially in patients with paroxysmal AF. Patients with persistent or long-standing persistent AF and with arrhythmia recurrence after previous PVI may benefit from additional ablation strategies, such as substrate modification of various forms or left atrial appendage isolation. New technologies and techniques, such as identification of the AF sources and magnetic resonance imaging-guided substrate modification, are on the way to further improve the success rates of catheter ablation for selected patients and might help to further reduce arrhythmia recurrence.

Conclusions: Pulmonary vein isolation is the treatment of choice for symptomatic patients with paroxysmal and persistent drug-refractory AF. The reconnection of previously isolated pulmonary veins remains the major cause of $\mathrm{AF}$ recurrence. Novel ablation tools, such as balloon technologies or alternative energy sources, might help to overcome this limitation. 
Patients with non-paroxysmal AF and with $\mathrm{AF}$ recurrence might benefit from alternative ablation strategies. However, further studies are warranted to further improve our knowledge of the underlying mechanisms of AF and to obtain long-term clinical outcomes on new ablation techniques.

Keywords: Ablation techniques; AF sources; Atrial fibrillation; Catheter ablation; Cryoballoon; Dielectric mapping; EPD; KODEX; Radiofrequency; Rotor ablation

\section{Key Summary Points}

The current state of the art and future perspectives of catheter ablation for the treatment of atrial fibrillation (AF) is reviewed and summarized.

Catheter ablation is a well-established treatment option for patients with symptomatic AF and is more successful at maintaining stable sinus rhythm than antiarrhythmic drugs.

Pulmonary vein isolation (PVI) is the treatment of choice for symptomatic patients with paroxysmal and persistent drug-refractory AF.

Patients with persistent or long-standing persistent $\mathrm{AF}$ and with arrhythmia recurrence after a previous PVI procedure may benefit from additional ablation strategies, such as substrate modification of various forms or left atrial appendage isolation.

New technologies and techniques, such as identification of AF sources and magnetic resonance imaging-guided substrate modification, are driving further improvements in the success rates of catheter ablation for selected patients and might help to further reduce arrhythmia recurrence.

\section{INTRODUCTION}

Atrial fibrillation (AF), the most common arrhythmia worldwide, is associated with increased morbidity and mortality [1, 2]. Catheter ablation of AF has evolved from an investigational procedure to the most effective treatment option for symptomatic patients, supported by encouraging clinical outcome data $[2,3]$. Catheter ablation for AF has been shown to be beneficial to patients with heart failure [4] and offers a significant improvement in the quality of life [5].

Multiple energy sources and a variety of ablation tools as well as different ablation strategies have been evaluated over the years $[6,7]$. In this review, we summarize the state of the art of catheter ablation for AF and the future prospectives of this procedure.

This article is based on previously conducted studies and does not contain any studies with human participants or animals performed by any of the authors.

\section{PATIENT SELECTION FOR CATHETER ABLATION AND RECOMMENDATIONS ACCORDING TO CURRENT GUIDELINES}

As demonstrated in a variety of published studies, the primary clinical benefit of catheter ablation of AF is improvement in the quality of life. Therefore, the primary selection criterion for AF ablation is the presence of symptoms, such as fatigue, palpitations and dyspnea. Current guidelines recommend that additional variables to be considered, including, among others, the presence of concomitant heart disease, obesity and sleep apnea, as these variables could result in a higher complication rate, as well as left atrial (LA) size, patient age and type of $\mathrm{AF}[2,8]$.

Catheter ablation of AF is recommended as a second-line therapy for patients with symptomatic paroxysmal AF (PAF) or persistent $\mathrm{AF}$ for whom therapy with antiarrhythmic drugs (AADs) (Class I) has failed and for patients with 
long-standing-persistent AF with a Class IIb indication. Although invasive cardiac procedures involve the potential for life-threatening complications, long-term AAD therapy has been shown to be more commonly associated with considerable side effects compared to ablation (17 vs. 8\%) [9]. Thus, novel guidelines also address the important issue of AF ablation as a first-line strategy in patients with symptomatic $\mathrm{PAF}$ or persistent $\mathrm{AF}$, prior to or in conjunction with Class I or III antiarrhythmic drug therapy (Class IIa) [2, 8].

\section{SUCCESS OF AF ABLATION}

The multiple-procedure success rate of pulmonary vein isolation (PVI) in patients with PAF after a 5-year follow-up period has been reported to be approximately $80 \%$, falling to about $60 \%$ after 10 years [10, 11]. For patients with persistent AF, stable sinus rhythm (SR) after successful PVI was reported in $25 \%$ after a single procedure and in $68 \%$ after multiple procedures, during a median follow-up of approximately 7 years [12].

However, estimation of the real success rate after catheter ablation of AF remains difficult due to inconsistencies in the definitions of procedural success and post-procedural recurrences, differences in post-procedural rhythm monitoring and differences in the analysis of outcomes after single or multiple procedures. To date, the electrophysiology community has defined AF recurrence as the occurrence of any symptomatic or asymptomatic atrial tachyarrhythmia after the procedure lasting for $>$ $30 \mathrm{~s}$. Newer studies have presented a novel definition focusing on the AF burden, which may represent a more relevant parameter for risk stratification and efficacy assessment after AF ablation [13, 14].

\section{FIRST STEP: PVI}

The pulmonary veins (PVs) have been shown to be the main trigger source for AF [15], and antral PVI as a stand-alone ablation strategy results in beneficial clinical outcomes as compared to medical therapy only [2].

Ablation strategies beyond PVI have not reproducibly been shown to be superior to PVIonly strategies in both paroxysmal and persistent AF [16-18]. The prospective and randomized Alster-Lost-AF trial assessed the outcome of two index strategies-stand-alone PVI and the stepwise approach of PVI followed by a substrate-modification approach involving complex fractionated atrial electrogram (CFAE) ablation and the creation of linear lesions-in patients with symptomatic, persistent or longstanding persistent AF. At 12 months, there were no significant differences in terms of freedom from atrial tachyarrhythmias between these two treatment strategies [16]. The CHASEAF clinical trial compared arrhythmia-free survival between patients with persistent AF undergoing PVI only and those undergoing a stepwise approach consisting of CFAE ablation and additional creation of linear lesions. This analysis also demonstrated no benefit for the stepwise approach [18]. Finally, the large multicenter STAR AF 2 trial compared PVI only and either PVI + CFAE ablation or PVI + creation of LA linear lesions as the index ablation strategy in patients with persistent AF; the results also did not demonstrate any benefit of the more complex ablation procedure [19].

Based on these results and others, PVI currently remains the only established endpoint for both patients with PAF and those with persistent $\mathrm{AF}$ and, according to the current $\mathrm{AF}$ guidelines, ablation beyond PVI is not recommended for patients with PAF or persistent AF undergoing a first ablation attempt $[2,20,21]$.

\section{Radiofrequency-Based AF Ablation-Still The Gold Standard?}

The most commonly used procedure for AF ablation is a point-by-point ablation via a single-tip catheter usually combined with a threedimensional (3D) mapping system, and the most common energy source is radiofrequency (RF) $[15,22]$. RF-based ablation in conjunction with an electro-anatomical mapping system (EAM) allows a significant reduction of 
fluoroscopy dosage and provides additional information, such as the LA activation pattern and LA voltage. Beyond that, this technology facilitates an optimized treatment of additional substrate, non-PV triggers or atrial tachycardia [23-25]. In general, a low complication rate is reported for RF-based catheter ablation, with the most common complications being pericardial effusion and tamponades and access-site complications, such as groin bleeding or arteriovenous fistula [22]. Although several attempts have been made to optimize navigation properties and to improve tip-to-tissue contact using robotic navigation [26-28], manually guided RF ablation is still the most widely used procedure.

Most of the established EAM systems use point-by-point acquisition of electrograms from a roving catheter with or without multi-electrode mapping capability $[29,30]$. To date, The Carto system (CARTO; Biosense Webster, Diamond Bar, CA, USA; Fig. 1) [6, 11] and the
EnSite NavX ${ }^{\mathrm{TM}}$ system (Endocardial Solutions, St. Jude Medical, Inc., St. Paul, MN, USA) have been the most detailed systems based on experience. Recently, a new mapping system (Rhythmia; Boston Scientific, Cambridge, MA, USA) that uses a small basket array of 64 electrodes (IntellaMap Orion; Boston Scientific) has been introduced with the aim to rapidly obtain an ultra-high-resolution EAM [31, 32]. Some clinical studies have already reported that the Rhythmia system in conjunction with the Orion catheter enables the determination of a successful PVI, may simplify ablation of complex atrial arrhythmias and could assist understanding of new targets for AF ablation [33-35].

Despite improvements in mapping technologies, the achievement of contiguous, transmural and permanent lesions using RFbased ablation remains challenging. Current technology is mainly limited by tip-to-tissue contact and the rather long learning curve of

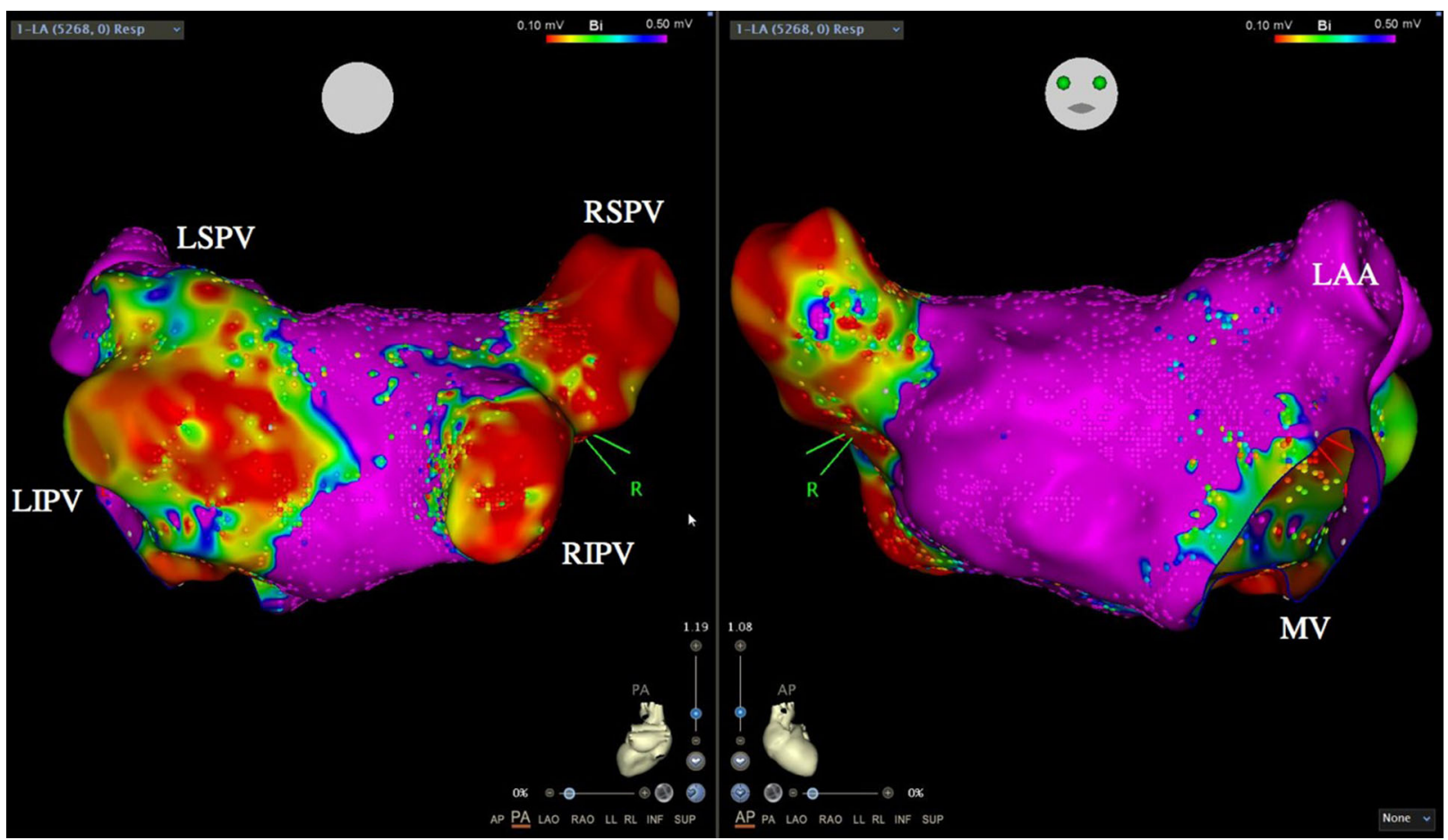

Fig. 1 Example of a left arterial voltage map acquired using the Carto system-guided high-density mapping in a posterior-anterior and anterior-posterior view, in a patient with atrial fibrillation recurrence after undergoing the pulmonary vein isolation (PVI) procedure. LSPV Left superior pulmonary vein, $L I P V$ left inferior pulmonary vein, $R S P V$ right superior pulmonary vein, $R I P V$ right inferior pulmonary vein, $L A A$ left atrial appendage, $M V$ mitral valve 
the operators [36, 37]. However, PV reconnection remains a dominant factor in arrhythmia recurrence after an initially successful catheter ablation, irrespective of the ablation device applied, and is substantially attributable to insufficient lesion formation during the index procedure [38]. Optimal lesion formation in RFbased ablation depends on a variety of procedural parameters, including power settings, catheter stability, ablation time, size of the catheter tip, temperature and cooling of the catheter tip and contact force (CF). Ablation using conventional catheters provides only limited information to predict sufficient lesion formation, such as diminution of the local electrogram or impedance drop.

The aim of recently developed CF-sensing technologies is to overcome, at least in part, these limitations. In clinical studies, the use of CF monitoring has been associated with improved procedural outcome, and low CF has been associated with acute procedural failure and sites of PV reconnection [36, 39]. However, improved clinical outcomes in CF-guided AF ablation has not been uniformly reproduced by other studies [40, 41].

The ablation index (AI) is a novel marker of ablation lesion quality which incorporates $\mathrm{CF}$, power and time in a weighted formula. The formula has been recently integrated into the automated lesion tagging software (VisiTag) of the 3D EAM system Carto 3 V4 (Biosense Webster Inc.). Previous clinical studies have confirmed the correlation between AI and PV reconnection rates [42] and reduced ablation duration [43] and have also shown that a prospective use of fixed AI targets results in a low rate of PV reconnection and high clinical success rates [44].

The limitations of catheter ablation using RF have led to further interest in the development of novel catheter designs and alternative energy sources for PVI. These include balloon-based ablation systems, such as the cryoballoon (CB) (ArcticFront; Medtronic, Inc., Minneapolis, MN, USA), the laserballoon (Heartlight ${ }^{\mathrm{TM}}$; CardioFocus, Marlborough, MA, USA), the RF Hot Balloon (Hayama Arrhythmia Institute, Kanagawa, Japan) and the "Globe" multi-electrode contact mapping and ablation system (Globe; Kardium Inc., Burnaby, BC, Canada).

\section{CB-Based PVI}

While RF-based PVI in combination with a 3D mapping system was long considered to be the "gold standard", the CB has emerged as the most commonly used alternative ablation tool for PVI and is now established as the second "gold standard" in patients with PAF in the current guidelines [2, 6] (Fig. 2).

CB-based AF ablation as a "single-shot" ablation modality is not only readily applicable and easy to learn, but is also, due to its "overthe-wire" strategy, a particularly safe tool. It is associated with a low incidence of major complications, specifically driven by a low number of pericardial effusions or tamponades [45]. In addition, the incidence of phrenic nerve palsy, which is the most common balloon-associated complication, can be kept considerably low by implementing safety algorithms, such as phrenic nerve pacing and monitoring of the compound motor action potential [6, 45]. Furthermore, the incidence of PV stenosis is a rare finding in patients undergoing $\mathrm{CB}$ ablation and has only been mentioned in incidental case reports $[46,47]$.

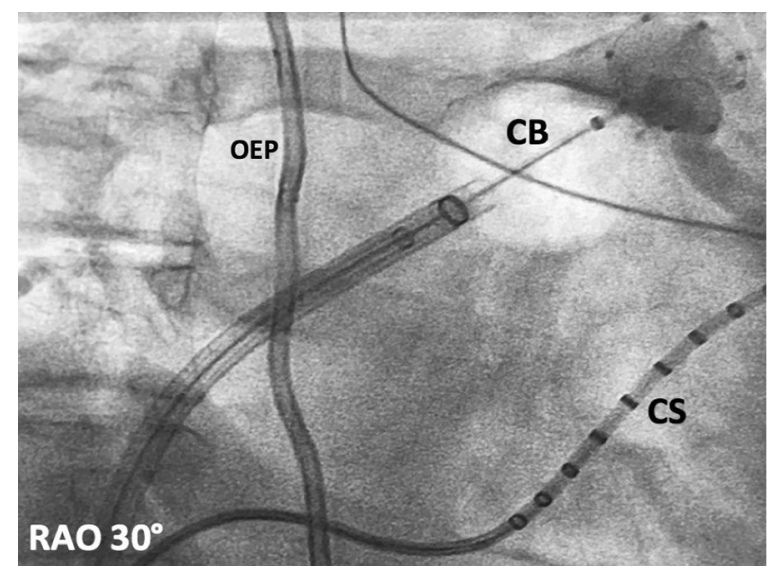

Fig. 2 Example of a cryoballoon ( $C B$ )-based PVI in a right-anterior-oblique $(R A O)$ view. The $\mathrm{CB}$ is located at the left-superior pulmonary vein (PV). Contrast medium shows total occlusion of this PV. CS Coronary sinus catheter, $O E P$ esophageal temperature probe 
Most data on CB ablation have been collected from patients suffering from PAF, with the data showing encouraging outcomes. The multicenter FIRE AND ICE trial, which prospectively randomized PAF patients to either RF- or CB-based PVI, demonstrated non-inferiority of $\mathrm{CB}$ ablation versus RF ablation in terms of efficacy and safety [6].

However, the positive findings from recent analyses encourage the application of CB-based catheter ablation to patients with persistent AF also. The recently published Cryo4persistent study focused not only on the recurrence of AF after previous CB-based PVI but also on the symptoms, and demonstrated a significant improvement in patients' quality of life following $\mathrm{CB}$ ablation in those with persistent $\mathrm{AF}$ [48]. These findings were confirmed by Mörtsell et al. who analyzed data from two large prospective registries (the AF Ablation LongTerm registry within the EURObservational Research Programme [AFA EORP] and the Swedish catheter ablation registry) [49]. These authors reported a lower EHRA (European Heart Rhythm Association) score-a measure of AFrelated symptoms-and a lower rate of continued antiarrhythmic drugs after CB-based AF ablation. These effects may not only have a positive impact on patients' quality of life, but also a positive impact on healthcare economics [49].

\section{Endoscopic Laserballoon-Based PVI}

The endoscopic ablation system (EAS; CardioFocus) is a balloon-based ablation system that incorporates a titratable laser energy source and a miniature $2 \mathrm{~F}$ endoscope that enables an endoscopic view into the target PV. The result is visually guided laserballoon ablation, which enables PVI under direct endoscopic-and thus direct visual-control. A number of smaller studies have demonstrated the feasibility of EAS-guided PVI with convincing acute efficacy and a favorable safety profile $[7,50]$. In addition, 1-year clinical follow-up data from a prospective multicenter study in patients with PAF showed a single-procedure clinical success rate of $63 \%$ in patients not receiving anti- arrhythmic medication [51]. These results are in agreement with those from an analysis performed by Dukkipati et al. who reported a 1 -year success rate in patients off anti-arrhythmic drugs of $60 \%$ after multiple procedures $[52,53]$.

The second-generation balloon system Heartlight $^{\mathrm{TM}}$ (Excalibur, CardioFocus), whose features include real-time balloon sizing for rapid engagement with all sizes and shapes of $\mathrm{PV}$, has become commercially available, as has the recently introduced third-generation balloon system Heartlight ${ }^{\mathrm{TM}}$ X3, (CardioFocus), which provides an automated laser source rotation to prevent gaps between applications and to further reduce application and procedure times.

Future modifications of this system may include electrodes on the balloon surface to provide real-time electrical recordings.

\section{RF Hotballoon-Based PVI}

The RF Hotballoon (Hayama Arrhythmia Institute, Kanagawa, Japan) consists of a compliant balloon (diameter $25-35 \mathrm{~mm}$ ) that is introduced into and manipulated within the left atrium via a $13 \mathrm{~F}$ steerable transseptal sheath. After verification of optimal PV-occlusion the inner fluid is heated up to $70-75^{\circ} \mathrm{C}$ via an RF generator. The assessment of initial studies consisted of a promising acute efficacy in combination with a satisfying safety profile, although a potentially higher risk of PV-stenosis was discussed by the authors [54]. In addition, some authors reported that a high rate of touch-up RF was required $[55,56]$.

\section{Globe Multi-electrode Mapping and Ablation System}

The novel "Globe" multi-electrode array catheter ablation system, which consists of 16 ribs with 122 gold-plated electrodes, is both a diagnostic and an RF ablation tool. A first-in-man study confirmed the feasibility of this system for single-shot PVI. Detailed information from several electrodes may enable voltage mapping with substrate modification and rotor mapping 
[57]. Further studies involving a larger number of patients are needed to show the efficacy of this ablation tool.

\section{CATHETER ABLATION OF AF BEYOND PVI}

Pulmonary vein isolation is an effective treatment strategy for patients with symptomatic PAF, but the success rate of this strategy remains limited in patients with persistent and long-standing persistent AF $[2,58]$. Additional ablation supplementary to PVI may be considered in patients with recurrent $\mathrm{AF}$ after the initial stand-alone PVI procedure or in patients who do not achieve restoration of stable sinus rhythm despite successful recurrent PVI [2]. To improve the success rate of catheter ablation, ablation strategies beyond PVI might be applied to treat additional arrhythmogenic substrates. The most commonly used strategies are ablation of CFAE and/or the creation of additional linear lesions in the left or right atrium [2].

Complex fractionated atrial electrograms, introduced by Nademanee et al. [59], are "low voltage atrial electrograms" (ranging from 0.04 to $0.25 \mathrm{mV}$ ), that have fractionated electrograms composed of two or more deflections and/or have a perturbation of the baseline with continuous deflection of a prolonged activation complex". Nademanee et al. reported that ablation of CFAE was efficacious in restoring SR in patients with PAF as well as in those with persistent $\mathrm{AF}$, with $87 \%$ of these patients being free of atrial arrhythmia recurrence after a mean follow-up of $839 \pm 493$ days [59]. This ablation strategy has since been applied in numerous clinical studies, generally in addition to PVI. All of these studies share the observation of a potential reduction of AF recurrence after a single procedure compared to stand-alone PVI. However, this benefit is overshadowed by a higher incidence of postprocedural atrial tachycardias, increased mean procedure times, mean RF energy application times and lack of reproducibility $[16,60]$.

Another innovative ablation strategy has recently been proposed by Narayan et al. [61] who hypothesized that ablation of AF drivers (so-called rotors or focal impulses) might result in improved and longer arrhythmia-free survival in patients with highly symptomatic PAF or persistent $\mathrm{AF}$ than would ablation of pulmonary and non-pulmonary vein triggers. To test this hypothesis, the authors conducted the CONFIRM trial (Conventional Ablation for Atrial Fibrillation With or Without Focal Impulse and Rotor Modulation), which enrolled 92 patients with highly symptomatic PAF or persistent $\mathrm{AF}$ in a two-arm 1:2 design with ablation of AF sources (Focal Impulse and Rotor Modulation [FIRM]-guided) followed by conventional ablation, mainly PVI $(n=36)$, or conventional ablation alone $(n=71$; FIRMblinded). Analysis of the data revealed that FIRM ablation at patient-specific sources could acutely terminate or slow the AF cycle length and improve clinical outcomes [61]. While the initial results and long-term data of the CONFIRM trial were promising, subsequent studies have shown inconsistent results [62]. Despite high operator-dependent variability, the fact that active and passive sources cannot be discriminated by this technology might play an additional role regarding the efficacy of AF driver elimination during FIRM-guided ablation $[63,64]$.

Electrographic flow (EGF) mapping has recently been introduced as a novel technology for the identification and characterization of $\mathrm{AF}$ sources in humans. Bellmann et al. demonstrated that EGF is the first method to have the potential to identify active AF sources during AF ablation and to be able to discriminate these from passive rotational phenomena, thereby possibly allowing for improved guidance of the AF ablation procedure $[65,66]$.

AF analysis using a non-invasive body surface mapping technique has also been shown to be able to identify reentrant and focal sources that play a potential role in driving and perpetuating AF. This non-invasive mapping-guided ablation strategy has been shown to be effective especially in patients with persistent AF [67, 68]. In 2017, Knecht et al. sought to evaluate the utility of non-invasive electrocardiogram (ECG) mapping as a practical tool prior to catheter ablation of persistent AF [68]. These authors performed ECG mapping ECVUE $^{\mathrm{TM}}$; 
CardioInsight Technologies, Medtronic, Dublin Ireland) in 118 patients with the aim to detect AF drivers. The CardioInsight Technologies noninvasive 3D mapping system collects chest ECG signals and merges these signals with computed tomography (CT) scan data in order to produce and display simultaneous biatrial maps that enable visualization of AF drivers. During the study, targeted drivers were ablated followed by PVI and left atrium linear lesion ablation. In the majority of patients, AF termination (into SR or atrial tachycardia) could be achieved only by ablation of the detected drivers, and ablation of these drivers resulted in favorable AF-free survival at the 1-year followup [68].

Local voltage abnormalities are also used as a surrogate for diseased cardiac tissue, such as atrial fibrosis. Recent studies using late gadolinium enhancement cardiac magnetic resonance imaging (MRI) have demonstrated that this diagnostic modality can facilitate the identification of structural changes in atrial tissue, thereby improving our understanding of the pathophysiology of AF. Moreover, extensive LA tissue fibrosis has shown to predict poor outcome after catheter ablation of AF [69]. Furthermore, imaging of atrial fibrosis using MRI has evolved to a tool that can be used to improve clinical outcomes after AF ablation by allowing a patient-specific, individualized approach [70]. In the ongoing DECAAF II study, patients will be randomized to receive conventional PVI or PVI + fibrosis-guided ablation. The primary outcome is defined as recurrence of AF. Results are expected to be released in July 2021 and will give further insights into the impact of fibrosis on AF ablation outcomes.

The left atrial appendage (LAA) has also been identified as a source of atrial arrhythmias as it can potentially trigger and sustain AF. Recently, the BELIEF trial showed that an electrical isolation of the LAA in addition to PVI could increase clinical success rates [71]. This same result was also reported by other investigators [72]. In addition to a more distinct ablation along the base of the LAA aiming at isolation $[71,72]$, some operators achieve LAA isolation by wide-area LA ablation with LA lines in addition to PVI [73]. Nevertheless, it should be kept in mind that isolation of the LAA in these cases has been shown to be associated with a significantly increased risk for LAA thrombus formation and thromboembolic events [74, 75]. Therefore, the indication for LAA isolation should be reserved for patients with highly symptomatic, long-standing persistent AF or patients with recurrent atrial tachyarrhythmia recurrence.

\section{FUTURE PERSPECTIVES IN AF ABLATION}

The aim of novel technologies is to improve the safety profile and clinical outcomes of AF ablation, reduce procedure time and fluoroscopy dosages, shorten the learning curve of the operators and possibly also help to improve our still limited understanding of the underlying mechanism of $\mathrm{AF}$, especially of persistent and long-standing persistent AF.

As mentioned in the above text, RF energy and cryoenergy are to date the two most widely used energy modalities for the treatment of cardiac arrhythmias. Thermal ablation, however, may lead to severe complications, such as PV stenosis and esophageal ulceration, despite several improvements to these technologies and applied safety algorithms [22, 45]. A novel technology, irreversible electroporation (IRE), may overcome these limitations. With IRE, a direct current is applied and the generated high electrical field produces pores in the phospholipid membranes of the cells, which leads to an irreversible breakdown of membrane structure and function and ultimately cell death. The first animal studies confirmed a significant effect of IRE on cardiac cells and therefore lesion formation as well as the safety of the system on surrounding intra- and extracardiac structures $[76,77]$. In vivo, clinical experiments are required to investigate the clinical implication of these observations.

A "High Power, Short Duration" strategy (HPSD) has recently been propagated as a further step towards the improvement of lesion quality during RF ablation in patients with symptomatic AF. Although this strategy is not absolutely new, it has been shown that HPSD 


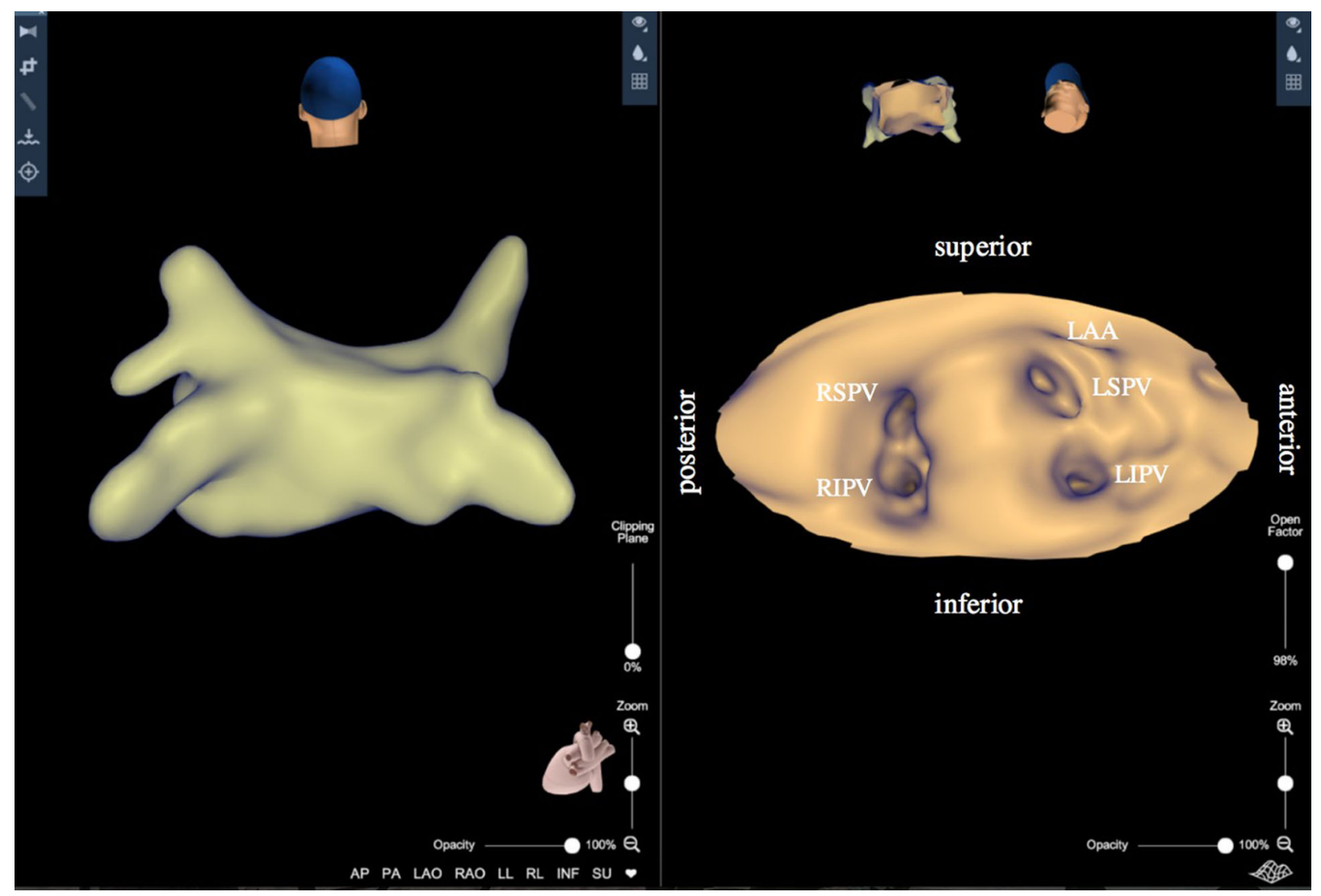

Fig. 3 The KODEX-EPD system provides the opportunity to visualize a cardiac chamber in a flattened panoramic view with the PANO view mode (posterior-anterior view) in addition to creating a three-dimensional map

ablation creates wider but more shallow lesions ex vivo as well as in vivo [78, 79], which could avoid damage to adjacent structures, such as the esophagus or the phrenic nerve, during ablation procedures. The feasibility and safety of HPSD strategy during AF ablation has already been confirmed in a clinical setting [80]. Additional advantages of this procedure are the possible decreases in procedure and fluoroscopy times, particularly when novel catheter technologies, such as the QDOT-FAST catheter, are used [81].

The HELIOSTAR RF Balloon Ablation Catheter (Biosense Webster, Inc.) has recently been introduced. It conforms to any pulmonary vein anatomy and has ten irrigated electrodes, which allows the operator to deliver different levels of energy during ablation. The device is compatible with the Biosense Webster CARTO 3 Mapping System (Biosense Webster, Inc.) and can therefore reduce radiation exposure during an ablation procedure. Its feasibility and safety profile as well as the clinical impact for the treatment of AF are still under evaluation. The STELLAR study (Safety and Effectiveness of the Multi-Electrode Radiofrequency Balloon Catheter for the Treatment of Symptomatic paroxysmal Atrial Fibrillation) is a pivotal, prospective, multicenter, single-arm clinical evaluation of the multi-electrode RF-balloon. Data are expected to be released 2021 .

Two other balloon ablation systems are under study, namely the POLARx cryo-ablation catheter (Boston Scientific) and the Apama RF hot balloon (Boston Scientific).

KODEX-EPD (KODEX-EPD ${ }^{\mathrm{TM}}$; EPD Solutions, Philips, Eindhoven, The Netherlands) is a novel cardiac imaging system providing electroanatomical 3D-visualization for guiding the treatment of PVI or complex atrial arrhythmias. It utilizes wide-band dielectric sensing and a 
unique bending of electric fields technology to generate high-resolution images of the cardiac anatomy. The acquisition process involves contact and non-contact mapping maneuvers using any type of diagnostic or ablation catheter. Anatomical mapping of the LA using KODEX-EPD bears the potential to create CTlike images without the need for additional periprocedural fluoroscopy [82]. In addition to the creation of a 3D anatomical shell, the KODEX-EPD system provides the opportunity to visualize a cardiac chamber in a flattened panoramic view with the PANO view mode (Fig. 3).

The results of the UNCOVER-AF (Utilizing Novel Dipole Density Capabilities to Objectively Visualize the Etiology of Rhythms in Atrial Fibrillation) trial have only very recently been published [83]. This prospective, nonrandomized trial was conducted at 13 centers in Europe and Canada and examined the use of a novel, non-contact imaging and mapping system that uses ultrasound to reconstruct atrial chamber anatomy and measures the timing and density of dipolar, ionic activation of the myocardium to guide ablation of atrial arrhythmias in patients with persistent AF. The results confirmed the feasibility and safety of this novel ultrasound imaging and charge density mapping system during AF ablation. The analysis also demonstrated a AF-free survival of 73 and 93\% after a single and second procedure after 12 months, respectively [83].

\section{CONCLUSIONS}

Catheter ablation is a well-established treatment option for patients with symptomatic AF and more effective at maintaining SR than antiarrhythmic drugs. Currently, the most effective technique for AF ablation is circumferential isolation of the PVs, irrespective of $\mathrm{AF}$ type. RF-based and $\mathrm{CB}$ ablation are equally effective in patients with PAF. Patients with persistent or long-standing persistent $\mathrm{AF}$ and with recurrence after an index ablation procedure might benefit from additional and more extensive ablation strategies. Novel technologies, such as KODEX-EPD and IRE, as well as other emerging techniques have the potential to improve the success rates of catheter ablation for select patient groups.

\section{ACKNOWLEDGEMENTS}

Funding. No funding or sponsorship was received for this study or for the publication of this article.

Authorship. All named authors meet the International Committee of Medical Journal Editors (ICMJE) criteria for authorship for this article, take responsibility for the integrity of the work as a whole, and have given their approval for this version to be published.

Disclosures. Andreas Metzner received speaker's honoraria and travel grants from Medtronic, Biosense Webster and Cardiofocus. Andreas Rillig received travel grants from Biosense, Medtronic, St. Jude Medical, CardioFocus, EP Solutions and Ablamap and lecture and consultant fees from St. Jude Medical, Medtronic, Biosense, CardioFocus, Novartis and Boehringer Ingelheim. Andreas Rillig is a member of the journal's Editorial Board. Laura Rottner, Barbara Bellmann, Tina Lin, Bruno Reissmann, Tobias Tönnis, Ruben Schleberger, Moritz Nies, Christiane Jungen, Leon Dinshaw, Niklas Klatt, Jannis Dickow, Paula Münkler and Christian Meyer have nothing to disclose.

Compliance with Ethics Guidelines. This article is based on previously conducted studies and does not contain any studies with human participants or animals performed by any of the authors.

Open Access. This article is distributed under the terms of the Creative Commons Attribution-NonCommercial 4.0 International License (http://creativecommons.org/licenses/ by-nc/4.0/), which permits any noncommercial use, distribution, and reproduction in any medium, provided you give appropriate credit to the original author(s) and the source, 
provide a link to the Creative Commons license, and indicate if changes were made.

\section{REFERENCES}

1. Feinberg WM, Blackshear JL, Laupacis A, Kronmal R, Hart RG. Prevalence, age distribution, and gender of patients with atrial fibrillation. Analysis and implications. Arch intern Med. 1995;155(5): 469-73.

2. Kirchhof P, Benussi S, Kotecha D, et al. 2016 ESC Guidelines for the management of atrial fibrillation developed in collaboration with EACTS. Europace. 2016;18(11):1609-78.

3. Packer DL, Mark DB, Robb RA, et al. Effect of catheter ablation vs antiarrhythmic drug therapy on mortality, stroke, bleeding, and cardiac arrest among patients with atrial fibrillation: The CABANA randomized clinical trial. JAMA. 2019;321:1261-74.

4. Marrouche NF, Kheirkhahan M, Brachmann J. Catheter ablation for atrial fibrillation with heart failure. N Engl J Med. 2018;379(5):492.

5. Blomstrom-Lundqvist C, Gizurarson S, Schwieler J, et al. Effect of catheter ablation vs antiarrhythmic medication on quality of life in patients with atrial fibrillation: the CAPTAF randomized clinical trial. JAMA. 2019;321(11):1059-68.

6. Kuck KH, Brugada J, Furnkranz A, et al. Cryoballoon or radiofrequency ablation for paroxysmal atrial fibrillation. N Engl J Med. 2016;374(23):2235-45.

7. Reissmann B, Budelmann T, Wissner E, et al. Fiveyear clinical outcomes of visually guided laser balloon pulmonary vein isolation for the treatment of paroxysmal atrial fibrillation. Clin Res Cardiol. 2018;107(5):405-12.

8. Calkins H, Hindricks G, Cappato R, et al. 2017 HRS/ EHRA/ECAS/APHRS/SOLAECE expert consensus statement on catheter and surgical ablation of atrial fibrillation. Heart Rhythm. 2017;14(10):e275-444.

9. Noheria A, Kumar A, Wylie JV Jr, Josephson ME. Catheter ablation vs antiarrhythmic drug therapy for atrial fibrillation: a systematic review. Arch Int Med. 2008;168(6):581-6.

10. Tilz RR, Heeger $\mathrm{CH}$, Wick A, et al. Ten-year clinical outcome after circumferential pulmonary vein isolation utilizing the Hamburg approach in patients with symptomatic drug-refractory paroxysmal atrial fibrillation. Circ Arrhythm Electrophysiol. 2018;11(2):e005250.

11. Ouyang F, Tilz R, Chun J, et al. Long-term results of catheter ablation in paroxysmal atrial fibrillation: lessons from a 5-year follow-up. Circulation. 2010;122(23):2368-77.

12. Brooks S, Metzner A, Wohlmuth $\mathrm{P}$, et al. Insights into ablation of persistent atrial fibrillation: lessons from 6-year clinical outcomes. J Cardiovasc Elektrophysiol. 2018;29(2):257-63.

13. Go AS, Reynolds K, Yang J, et al. Association of burden of atrial fibrillation with risk of ischemic stroke in adults with paroxysmal atrial fibrillation: the KP-RHYTHM study. JAMA Cardiol. 2018;3(7): $601-8$.

14. Kuck KH, Brugada J, Schluter $M$, et al. The FIRE AND ICE trial: what we know, what we can still learn, and what we need to address in the future. J Am Heart Assoc. 2018;7(24):e010777.

15. Haissaguerre M, Jais $\mathrm{P}$, Shah DC, et al. Spontaneous initiation of atrial fibrillation by ectopic beats originating in the pulmonary veins. N Engl J Med. 1998;339(10):659-66.

16. Fink T, Schluter $\mathrm{M}$, Heeger $\mathrm{CH}$, et al. Stand-alone pulmonary vein isolation versus pulmonary vein isolation with additional substrate modification as index ablation procedures in patients with persistent and long-standing persistent atrial fibrillation: the randomized Alster-Lost-AF Trial (Ablation at St. Georg Hospital for Long-Standing Persistent Atrial Fibrillation). Circ Arrhythm Electrophysiol. 2017;10(7):e005114.

17. Verma A, Jiang CY, Betts TR, et al. Approaches to catheter ablation for persistent atrial fibrillation. N Engl J Med. 2015;372(19):1812-22.

18. Vogler J, Willems S, Sultan A, et al. Pulmonary vein isolation versus defragmentation: the CHASE-AF clinical trial. J Am Coll Cardiol. 2015;66(24): 2743-52.

19. Verma A, Mantovan R, Macle L, et al. Substrate and trigger ablation for reduction of atrial fibrillation (STAR AF): a randomized, multicentre, international trial. Eur Heart J. 2010;31(11):1344-56.

20. Arbelo E, Guiu E, Ramos P, et al. Benefit of left atrial roof linear ablation in paroxysmal atrial fibrillation: a prospective, randomized study. J Am Heart Assoc. 2014;3(5):e000877.

21. Mun HS, Joung B, Shim J, et al. Does additional linear ablation after circumferential pulmonary vein isolation improve clinical outcome in patients 
with paroxysmal atrial fibrillation? Prospective randomised study. Heart. 2012;98(6):480-4.

22. Cappato R, Calkins H, Chen SA, et al. Updated worldwide survey on the methods, efficacy, and safety of catheter ablation for human atrial fibrillation. Circ Arrhythm Electrophysiol. 2010;3(1): 32-8.

23. Ouyang F, Ernst S, Chun J, et al. Electrophysiological findings during ablation of persistent atrial fibrillation with electroanatomic mapping and double Lasso catheter technique. Circulation. 2005;112(20):3038-48.

24. Powell BD, Packer DL. Does image integration improve atrial fibrillation ablation outcomes, or are other aspects of the ablation the key to success? Europace. 2009;11(8):973-4.

25. Kistler PM, Rajappan K, Harris S, et al. The impact of image integration on catheter ablation of atrial fibrillation using electroanatomic mapping: a prospective randomized study. Eur Heart J. 2008;29(24):3029-36.

26. Rillig A, Lin T, Schmidt B, et al. Experience matters: long-term results of pulmonary vein isolation using a robotic navigation system for the treatment of paroxysmal atrial fibrillation. Clin Res Cardiol. 2016;105(2):106-16.

27. Rillig A, Schmidt B, Di Biase L, et al. Manual versus robotic catheter ablation for the treatment of atrial fibrillation: the man and machine trial. JACC Clin Electrophysiol. 2017;3(8):875-83.

28. Maurer T, Sohns C, Deiss S, et al. Significant reduction in procedure duration in remote magneticguided catheter ablation of atrial fibrillation using the third-generation magnetic navigation system. J Interv Cardiac Electrophysiol. 2017;49(3):219-26.

29. Weerasooriya R, Jais P, Wright M, et al. Catheter ablation of atrial tachycardia following atrial fibrillation ablation. J Cardiovasc Electrophysiol. 2009;20(7):833-8.

30. Maille B, Das M, Hussein A, et al. Accuracy of left atrial bipolar voltages obtained by ConfiDENSE multielectrode mapping in patients with persistent atrial fibrillation. J Cardiovasc Eelectrophysiol. 2018;29(6):881-8.

31. Ptaszek LM, Chalhoub F, Perna F, et al. Rapid acquisition of high-resolution electroanatomical maps using a novel multielectrode mapping system. J Interven Cardiac Electrophysiol. 2013;36(3): 233-42.

32. Sohns C, Saguner AM, Lemes C, et al. First clinical experience using a novel high-resolution electroanatomical mapping system for left atrial ablation procedures. Clin Res Cardiol. 2016;105: 992-1002.

33. Anter E, Tschabrunn CM, Contreras-Valdes FM, Li J, Josephson ME. Pulmonary vein isolation using the Rhythmia mapping system: verification of intracardiac signals using the Orion mini-basket catheter. Heart Rhythm. 2015;12(9):1927-34.

34. Ernst S, Saenen J, Rydman R, et al. Utility of noninvasive arrhythmia mapping in patients with adult congenital heart disease. Card Electrophysiol Clin. 2015;7(1):117-23.

35. Rottner L, Metzner A, Ouyang F, et al. Direct comparison of point-by-point and rapid ultra-high-resolution electroanatomical mapping in patients scheduled for ablation of atrial fibrillation. J Cardiovasc Electrophysiol. 2017;28(3):289-97.

36. Kautzner J, Neuzil P, Lambert H, et al. EFFICAS II: optimization of catheter contact force improves outcome of pulmonary vein isolation for paroxysmal atrial fibrillation. Europace. 2015;17(8): 1229-35.

37. Neuzil P, Reddy VY, Kautzner J, et al. Electrical reconnection after pulmonary vein isolation is contingent on contact force during initial treatment: results from the EFFICAS I study. Circ Arrhythm Electrophysiol. 2013;6(2):327-33.

38. Cheema A, Dong J, Dalal D, et al. Incidence and time course of early recovery of pulmonary vein conduction after catheter ablation of atrial fibrillation. J Cardiovasc Electrophysiol. 2007;18(4): 387-91.

39. Reddy VY, Shah D, Kautzner J, et al. The relationship between contact force and clinical outcome during radiofrequency catheter ablation of atrial fibrillation in the TOCCATA study. Heart Rhythm. 2012;9(11):1789-95.

40. Makimoto H, Lin T, Rillig A, et al. In vivo contact force analysis and correlation with tissue impedance during left atrial mapping and catheter ablation of atrial fibrillation. Circ Arrhythm Electrophysiol. 2014;7(1):46-54.

41. Stabile G, Solimene F, Calo L, et al. Catheter-tissue contact force values do not impact mid-term clinical outcome following pulmonary vein isolation in patients with paroxysmal atrial fibrillation. J Interv Card Electrophysiol. 2015;42(1):21-6.

42. Das M, Loveday JJ, Wynn GJ, et al. Ablation index, a novel marker of ablation lesion quality: prediction of pulmonary vein reconnection at repeat electrophysiology study and regional differences in target values. Europace. 2017;19(5):775-83. 
43. Santoro F, Metzner A, Brunetti ND, et al. Left atrial anterior line ablation using ablation index and inter-lesion distance measurement. Clin Res Cardiol. 2019;108(9):1009-16

44. Hussein A, Das M, Riva S, et al. Use of ablation index-guided ablation results in high rates of durable pulmonary vein isolation and freedom from arrhythmia in persistent atrial fibrillation patients. Circ Arrhythm Electrophysiol. 2018;11(9):e006576.

45. Rottner L, Fink $\mathrm{T}$, Heeger $\mathrm{CH}$, et al. Is less more? Impact of different ablation protocols on periprocedural complications in second-generation cryoballoon based pulmonary vein isolation. Europace. 2017;20:1459-67.

46. Thomas D, Katus HA, Voss F. Asymptomatic pulmonary vein stenosis after cryoballoon catheter ablation of paroxysmal atrial fibrillation. J Electrocardiol. 2011;44(4):473-6.

47. Tokutake K, Tokuda M, Ogawa T, Matsuo S, Yoshimura M, Yamane T. Pulmonary vein stenosis after second-generation cryoballoon ablation for atrial fibrillation. Heart Rhythm Case Rep. 2017;3(1): 36-9.

48. Boveda SMA, Nguyen DQ, Chun KRJ, et al. Singleprocedure outcomes and quality-of-life improvement 12 months post-cryoballoon ablation in persistent atrial fibrillation results from the multicenter CRYO4PERSISTENT AF trial. JACC Clin Electrophysiol. 2018;11:1440-7.

49. Mortsell D, Arbelo E, Dagres N, et al. Cryoballoon vs. radiofrequency ablation for atrial fibrillation: a study of outcome and safety based on the ESCEHRA atrial fibrillation ablation long-term registry and the Swedish catheter ablation registry. Europace. $2019 ; 21(4): 581-9$.

50. Wissner E, Deiss S, Kuck KH. Balloon-based pulmonary vein isolation for the treatment of paroxysmal atrial fibrillation (cryoballoon and laserballoon). Fut Cardiol. 2015;11(6):663-71.

51. Metzner A, Wissner E, Schmidt B, et al. Acute and long-term clinical outcome after endoscopic pulmonary vein isolation: results from the first prospective, multicenter study. J Cardiovasc Electrophysiol. 2013;24(1):7-13.

52. Dukkipati SR, Kuck KH, Neuzil P, et al. Pulmonary vein isolation using a visually guided laser balloon catheter: the first 200-patient multicenter clinical experience. Circ Arrhythm Electrophysiol. 2013;6(3):467-72.

53. Schmidt B, Neuzil P, Luik A, et al. Laser balloon or wide-area circumferential irrigated radiofrequency ablation for persistent atrial fibrillation: a multicenter prospective randomized study. Circ Arrhythm Ectrophysiol. 2017;10(12):e005767.

54. Yamaguchi Y, Sohara H, Takeda H, et al. Long-term results of radiofrequency hot balloon ablation in patients with paroxysmal atrial fibrillation: safety and rhythm outcomes. J Cardiovasc Electrophysiol. 2015;26(12):1298-306.

55. Nagashima K, Okumura Y, Watanabe I, et al. Hot balloon versus cryoballoon ablation for atrial fibrillation: lesion characteristics and middle-term outcomes. Circ Arrhythm Electrophysiol. 2018;11(5):e005861.

56. Wakamatsu Y, Nagashima K, Nakahara S, et al. Electrophysiologic and anatomic factors predictive of a need for touch-up radiofrequency application for complete pulmonary vein isolation: comparison between hot balloon- and cryoballoon-based ablation. J Cardiovasc Electrophysiol. 2019;30(8): $1261-69$

57. Kottkamp H, Moser F, Rieger A, Schreiber D, Ponisch C, Trofin M. Global multielectrode contact mapping plus ablation with a single catheter: preclinical and preliminary experience in humans with atrial fibrillation. J Cardiovasc Electrophysiol. 2017;28(11):1247-56.

58. Lemes C, Wissner E, Lin T, et al. One-year clinical outcome after pulmonary vein isolation in persistent atrial fibrillation using the second-generation $28 \mathrm{~mm}$ cryoballoon: a retrospective analysis. Europace. $2016 ; 18(2): 201-5$.

59. Nademanee K, Schwab MC, Kosar EM, et al. Clinical outcomes of catheter substrate ablation for highrisk patients with atrial fibrillation. J Am Coll Cardiol. 2008;51(8):843-9.

60. Wu SH, Jiang WF, Gu J, et al. Benefits and risks of additional ablation of complex fractionated atrial electrograms for patients with atrial fibrillation: a systematic review and meta-analysis. Int J Cardiol. 2013;169(1):35-43.

61. Narayan SM, Krummen DE, Shivkumar K, Clopton P, Rappel WJ, Miller JM. Treatment of atrial fibrillation by the ablation of localized sources: CONFIRM (conventional ablation for atrial fibrillation with or without focal impulse and rotor modulation) trial. J Am Coll Cardiol. 2012;60(7):628-36.

62. Mohanty S, Gianni C, Trivedi C, et al. Impact of rotor ablation in non-paroxysmal AF patients: findings from the per-protocol population of the OASIS trial at long-term follow-up. Am Heart J. 2018;205:145-8.

63. Gianni C, Mohanty S, Di Biase L, et al. Acute and early outcomes of focal impulse and rotor 
modulation (FIRM)-guided rotors-only ablation in patients with nonparoxysmal atrial fibrillation. Heart Rhythm. 2016;13(4):830-5.

64. Steinberg JS, Shah Y, Bhatt A, et al. Focal impulse and rotor modulation: acute procedural observations and extended clinical follow-up. Heart Rhythm. 2017;14(2):192-7.

65. Bellmann B, Lin T, Ruppersberg P, et al. Identification of active atrial fibrillation sources and their discrimination from passive rotors using electrographical flow mapping. Clin Res Cardiol. 2018;107(11):1021-32.

66. Bellmann B, Zettwitz M, Lin T, et al. Velocity characteristics of atrial fibrillation sources determined by electrographic flow mapping before and after catheter ablation. Int J Cardiol. 2019;286: 56-60.

67. Haissaguerre $\mathrm{M}$, Hocini $\mathrm{M}$, Shah $\mathrm{AJ}$, et al. Noninvasive panoramic mapping of human atrial fibrillation mechanisms: a feasibility report. J Cardiovasc Electrophysiol. 2013;24(6):711-7.

68. Knecht S, Sohal M, Deisenhofer I, et al. Multicentre evaluation of non-invasive biatrial mapping for persistent atrial fibrillation ablation: the AFACART study. Europace. 2017;19(8):1302-9.

69. Marrouche NF, Wilber D, Hindricks G, et al. Association of atrial tissue fibrosis identified by delayed enhancement MRI and atrial fibrillation catheter ablation: the DECAAF study. JAMA. 2014;311(5): 498-506.

70. Jadidi AS, Lehrmann H, Keyl C, et al. Ablation of persistent atrial fibrillation targeting low-voltage areas with selective activation characteristics. Circ Arrhythm Electrophysiol. 2016;9(3):e002962.

71. Di Biase L, Burkhardt JD, Mohanty P, et al. Left atrial appendage isolation in patients with longstanding persistent af undergoing catheter ablation: BELIEF trial. J Am Coll Cardiol. 2016;68(18): 1929-40.

72. Yorgun H, Canpolat U, Kocyigit D, Coteli C, Evranos B, Aytemir K. Left atrial appendage isolation in addition to pulmonary vein isolation in persistent atrial fibrillation: one-year clinical outcome after cryoballoon-based ablation. Europace. 2017;19(5): 758-68.

73. Heeger $\mathrm{CH}$, Rillig A, Geisler D, et al. Left atrial appendage isolation in patients not responding to pulmonary vein isolation. Circulation. 2019;139(5): 712-5.

74. Rillig A, Tilz RR, Lin $\mathrm{T}$, et al. Unexpectedly high incidence of stroke and left atrial appendage thrombus formation after electrical isolation of the left atrial appendage for the treatment of atrial tachyarrhythmias. Circ Arrhythm Electrophysiol. 2016;9(5):e003461.

75. Heeger $\mathrm{CH}$, Rillig A, Lin $\mathrm{T}$, et al. Feasibility and clinical efficacy of left atrial ablation for the treatment of atrial tachyarrhythmias in patients with left atrial appendage closure devices. Heart Rhythm. 2015;12(7):1524-31.

76. Wittkampf FHM, van Es R, Neven K. Electroporation and its relevance for cardiac catheter ablation. JACC Clin Electrophysiol. 2018;4(8):977-86.

77. Neven K, van Es R, van Driel V, et al. Acute and long-term effects of full-power electroporation ablation directly on the porcine esophagus. Circ Arrhythm Electrophysiol. 2017;10(5):e004672.

78. Bhaskaran A, Chik W, Pouliopoulos J, et al. Five seconds of 50-60 W radio frequency atrial ablations were transmural and safe: an in vitro mechanistic assessment and force-controlled in vivo validation. Europace. 2017;19(5):874-80.

79. Leshem E, Zilberman I, Tschabrunn CM, et al. Highpower and short-duration ablation for pulmonary vein isolation: biophysical characterization. JACC Clin Electrophysiol. 2018;4(4):467-79.

80. Winkle RA, Moskovitz R, Hardwin Mead R, et al. Atrial fibrillation ablation using very short duration $50 \mathrm{~W}$ ablations and contact force sensing catheters. J Interv Card Electrophysiol. 2018;52(1):1-8.

81. Reddy VY, Grimaldi M, De Potter T, et al. Pulmonary vein isolation with very high power, short duration, temperature-controlled lesions: the QDOT-FAST trial. JACC Clin Electrophysiol. 2019;5(7):778-86.

82. Maurer T, Kuck K-H, Schlüter M, et al. First clinical experience in high-resolution imaging of left atrial anatomy using a novel wide-band dielectric mapping system. Clin Res Cardiol 2019;108[Suppl 1].

83. Willems S, Verma A, Betts T, et al. Targeting nonpulmonary vein sources in persistent atrial fibrillation identified by noncontact charge density mapping (UNCOVER-AF trial). Circ Arrhythm Electrophysiol. 2019;12:e007233. 